\title{
Simulation Study on the Minimization of Moiré Pattems Caused by Microlens Array Films for Backlight Applications
}

\author{
Byung-Yun $\mathrm{Joo}^{1}$ and Jae-Hyeon $\mathrm{Ko}^{2}$ * \\ ${ }^{I}$ Department of Mechanical Engineering, Korea University of Technology and Education, \\ Cheonan 330-708, Korea \\ ${ }^{2}$ Department of Physics, Hallym University, Chuncheon 200-702, Korea
}

(Received August 8, 2014 : revised September 19, 2014 : accepted September 23, 2014)

\begin{abstract}
Microlens array (MLA) films have been used as brightness-enhancement components in many illumination systems, such as a backlight, which should meet the industrial needs of low cost and high brightness performance. A hexagonally-arranged MLA (HA-MLA) was chosen due to its high filling factor, which was a prerequisite for achieving high brightness-enhancement capability. The moiré fringes produced in a film stack composed of two HA-MLA were investigated, under four different lens configurations, by optical simulation. It was found that randomizing the lens configuration, while keeping the filling factor high, was effective way to develop high-gain MLA films for backlight applications.
\end{abstract}

Keywords : Moiré, Micro lens array, Backlight, Optical simulation

OCIS codes : (120.2040) Displays; (150.2950) Illumination

\section{INTRODUCTION}

Optical films based on the microlens array (MLA) technology are widely used for brightness enhancement in illumination systems [1]. For example, backlights for liquid crystal displays usually adopt one or two MLA films, in addition to prism films and/or a reflective polarizer, to achieve low power consumption [2-3]. In general, MLA films consist of randomly positioned hemispherical lenses, the filling factor of which is in the range of $65-70 \%$. The luminance gain of the MLA film is greater than that of a light diffuser film, but less than that of a one-dimensional prism film. The filling factor is defined as the ratio of the projection area of the lenses to the entire surface area of the film. A schematic structure of a conventional MLA film is shown in Table 1 (denoted as "conventional type"). Hemispherical microtextures $50-60 \mu \mathrm{m}$ in diameter are randomly arranged with a filling factor of $65-70 \%$ on the MLA film of this type. It is known that the brightnessenhancement performance of an MLA film can be enhanced by increasing the filling factor of its microlenses $[4,5]$.

Recently, optical films based on a hexagonally-arranged microlens array (HA-MLA) have attracted great attention because of their high potential for achieving better brightness- enhancement capability and lower production cost compared to conventional MLA films [6]. As compared in Table 1, the filling factor of the HA-MLA is around $70-80 \%$, which is higher than that of the conventional film. The upper limit of the filling factor $R_{\text {Filling }}$ for a HA-MLA is calculated to be $90.7 \%$ according to Eq. (1), when the diameter of the microlens $2 r$ is equal to the pitch $a$.

$$
R_{\text {Filing }}=\frac{\pi}{2 \sqrt{3}}\left(\frac{2 r}{a}\right)^{2} \times 100(\%)
$$

In addition, the hemispherical lens can be switched to an aspheric lens, which can be optimized for better brightnessenhancement capability [7, 8]. It is known that adopting one HA-MLA film in a backlight improves the on-axis brightness by approximately $8-10 \%$, compared to a conventional MLA film. Also, low production cost can be realized, because the amount of resin used in the manufacturing process can be reduced by decreasing lens diameter to 25-30 $\mu \mathrm{m}$. However, an HA-MLA may bring about moiré problems in a doubleor multiple-layer stack, due to the periodic modulation of the microlenses on the film substrate. The moire phenomenon degrades the picture quality of a display and thus should

\footnotetext{
*Corresponding author: hwangko@hallym.ac.kr

Color versions of one or more of the figures in this paper are available online.
} 
TABLE 1. Comparison of two microlens array structures: conventional randomly-arranged type versus hexagonally-arranged type

\begin{tabular}{|c|c|c|}
\hline & $\begin{array}{l}\text { Micro lens array film } \\
\text { (Conventional type) }\end{array}$ & $\begin{array}{c}\text { Micro lens array film } \\
\text { (Hexagonally-arranged type) }\end{array}$ \\
\hline \multicolumn{3}{|l|}{ Schematic } \\
\hline Texture arrangement & $\begin{array}{l}\text { Random arrangement } \\
\text { (Fill factor, } 65 \sim 70 \% \text { ) }\end{array}$ & $\begin{array}{c}\text { Hexagonal arrangement } \\
\text { (Fill factor, } 78 \% \text {, theoretically } 90.6 \% \text { ) }\end{array}$ \\
\hline Texture size & $50 \sim 60 \mu \mathrm{m}$, hemisphere & $25 \sim 30 \mu \mathrm{m}$, conic asphere \\
\hline Manufacturing cost & - & $\begin{array}{c}\text { Relatively low } \\
\text { (Small volume of resin material) }\end{array}$ \\
\hline Brightness & - & $\begin{array}{l}6 \sim 8 \% \text { enhanced } \\
\text { (High filling factor) }\end{array}$ \\
\hline Moire in the film stack & None & Severe moiré under certain conditions \\
\hline & & $\overline{2 \mathrm{~mm}}$ \\
\hline
\end{tabular}

be avoided in such applications. Much effort has been spent to analyze and reduce moiré phenomena $[1,9,10]$. One approach toward a moiré-free platform is to randomize the periodic microstructures to some degree [11].

In this respect, it is necessary to optimize the optical structure of an MLA film to remove or reduce the moiré phenomenon in an optical device such as a backlight. Usually 1-3 MLA films are adopted in a backlight, and a moiré pattern may be formed when the number of the MLA films is at least two. In this study, several approaches for avoiding moirés between two HA-MLA films were investigated by optical simulation based on the ray-tracing technique [12]. In general, moire formation between the MLA and the LCD pixel array is regarded as a more serious problem in LCD. Although the interference effect between the MLA and the LCD pixel array is not discussed in detail in this study, we do suggest a possibility for reducing this problem based on our approach.

\section{SIMULATION}

Figure 1 shows the schematic view of the MLA geometry adopted in the present moiré simulation. This model consists of two MLA films, the upper and the lower. The distance between upper and lower films was $0.15 \mathrm{~mm}$, which was determined by considering the thickness of the base film (usually $0.125 \mathrm{~mm}$ ) and the height of the MLA (10-30 $\mu \mathrm{m})$. Circular holes with a diameter of $28 \mu \mathrm{m}$ were hexagonally-arranged on the upper film at a pitch of 30

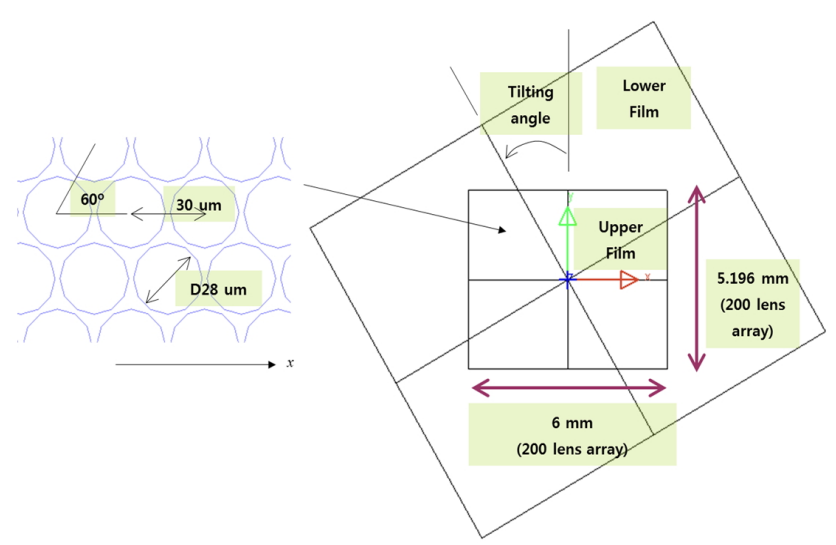

FIG. 1. Schematic diagram of the model geometry for the moiré simulation.

$\mu \mathrm{m}$ along the $x$ direction. The dimension of the upper film was $6.000 \times 5.196 \mathrm{~mm}^{2}$, on which $200 \times 200$ holes were arranged. The moiré fringe is recognized generally when the moiré period is larger than $0.5 \mathrm{~mm}$. Therefore, the present observation area of $6.000 \times 5.196 \mathrm{~mm}^{2}$ seems large enough for scrutinizing the moiré problem. The lower film was placed beneath the upper film. The area of the lower film was $12.0 \times 10.2 \mathrm{~mm}^{2}$, and it was tilted with respect to the upper film by a certain angle. The hole texture of the lower film was prepared according to four possible approaches to reducing the moiré pattern, while the hole arrangement of the upper film was fixed. The four simulation conditions are summarized in Table 2.

The first approach adopts the same hexagonally-arranged 
TABLE 2. Simulation conditions: four possible approaches for avoiding moirés

\begin{tabular}{|c|c|c|c|c|}
\hline No. & $\begin{array}{l}\text { Possible approaches for } \\
\text { avoiding moiré pattern }\end{array}$ & $\begin{array}{l}\text { Simulation conditions } \\
\text { for the upper film }\end{array}$ & $\begin{array}{l}\text { Simulation conditions } \\
\text { for the lower film }\end{array}$ & $\begin{array}{l}\text { Schematic view of the } \\
\text { lower film }\end{array}$ \\
\hline 1 & $\begin{array}{l}\text { Tilting the lower film with } \\
\text { respect to the upper film }\end{array}$ & & $\begin{array}{l}\text { Pitch }=30 \mu \mathrm{m} \\
\text { Dia. }=28 \mu \mathrm{m} \\
\text { Hexagonal } \\
\text { FF }=79 \% \\
\text { Tilt angle resolution }=2^{\circ}\end{array}$ & \\
\hline 2 & $\begin{array}{l}\text { Varying the hole } \\
\text { diameter and the pitch of the } \\
\text { lower film }\end{array}$ & \multirow{3}{*}{$\begin{array}{l}\text { Pitch }=30 \mu \mathrm{m} \\
\text { Dia. }=28 \mu \mathrm{m} \\
\text { Hexagonal } \\
\mathrm{FF}=79 \%\end{array}$} & $\begin{array}{l}\text { Pitch }=28,26,24,22,20,18, \\
16,15 \mu \mathrm{m} \\
\text { Dia.=Pitch-2 } \mu \mathrm{m} \\
\text { Hexagonal } \\
\mathrm{FF}=78,77,76,74,73,71, \\
69,68 \%\end{array}$ & \\
\hline 3 & $\begin{array}{l}\text { Randomizing the hole } \\
\text { diameter of the lower film } \\
\text { and tilting it with respect to } \\
\text { the upper film }\end{array}$ & & $\begin{array}{l}\text { Pitch }=30 \mu \mathrm{m} \\
\text { Dia. }=20 \sim 30 \mu \mathrm{m} \\
\text { Hexagonal } \\
\mathrm{FF}=63 \\
\text { Tilt angle resolution }=2^{\circ}\end{array}$ & \\
\hline 4 & $\begin{array}{l}\text { Randomizing the hole } \\
\text { diameter of the lower film } \\
\text { based on the sine shift } \\
\text { algorithm }\end{array}$ & & $\begin{array}{l}\text { Pitch }=28 \mu \mathrm{m} \\
\text { Minimum gap between } \\
\text { dots }=1 \mu \mathrm{m} \\
\text { Random } \\
\mathrm{FF}=70 \% \\
\text { Tilt angle resolution }=2^{\circ}\end{array}$ & \\
\hline
\end{tabular}

* FF=Filling Factor

hole texture as that of the upper film, and only the relative orientation was changed systematically, i.e. the lower film was tilted with respect to the upper film at a step of $2^{\circ}$. In the second approach the pitch between the holes of the lower film was changed from $15 \mu \mathrm{m}$ to $28 \mu \mathrm{m}$. The diameter of each hole was set to be smaller than the pitch by $2 \mu \mathrm{m}$, so that the fill factor was varied from 68 to $78 \%$. The third approach was to randomize the hole size of the lower film in the range of $20-30 \mu \mathrm{m}$ in diameter, and to tile the film with respect to the upper film. Finally, in the fourth approach the hole size was fixed to be $28 \mu \mathrm{m}$ and the holes were randomly arranged using the sine shift algorithm [13].

During the simulation collimated light rays were incident upon the film stack from the bottom side of the lower film, and the transmitted rays were gathered by a receiver located at the top of the upper film. The resolution of the receiver (i.e. the mesh size) was $3.00 \times 2.58 \mu^{2}$. The transmittance of the holes was set to be $100 \%$ and the surface other than the holes absorbed the incident light completely. Commercially available software (LightTools, ORA Co.) was used as the ray-tracing simulator.

\section{RESULTS AND DISCUSSION}

Figure 2 shows the simulation results for the first approach (denoted as "approach 1" in Table 2), where two films with the same hole texture were tilted relative to each other at a certain angle. Fig. 2(a) shows that there is no moiré fringe when the tilt angle of the lower film is $0^{\circ}$, which means the moiré period is infinite. However, as Fig. 2(b) shows, severe moiré fringes occur even at a very small tilt angle such as $2^{\circ}$, due to the long period of the moiré. The moiré pattern becomes weaker as the tilt angle increases, until an angle of $\sim 24^{\circ}$, where the moiré fringes are almost invisible. Upon further increasing the tilt angle beyond $24^{\circ}$ the moiré pattern becomes stronger, and then it finally disappears again at an angle of $60^{\circ}$. Since the hexagonal arrangement repeats every $60^{\circ}$, the moiré pattern also disappears at every $60^{\circ}$, when the hole arrangements of both films match. However, these angles cannot be optimal, because the allowable tolerance of the tilt angle is very small. Instead, the tilt angle of $24^{\circ}$ is favorable, from the view point of tolerance. However, another moiré pattern might form due to interference between the MLA film and the LCD pixels, 


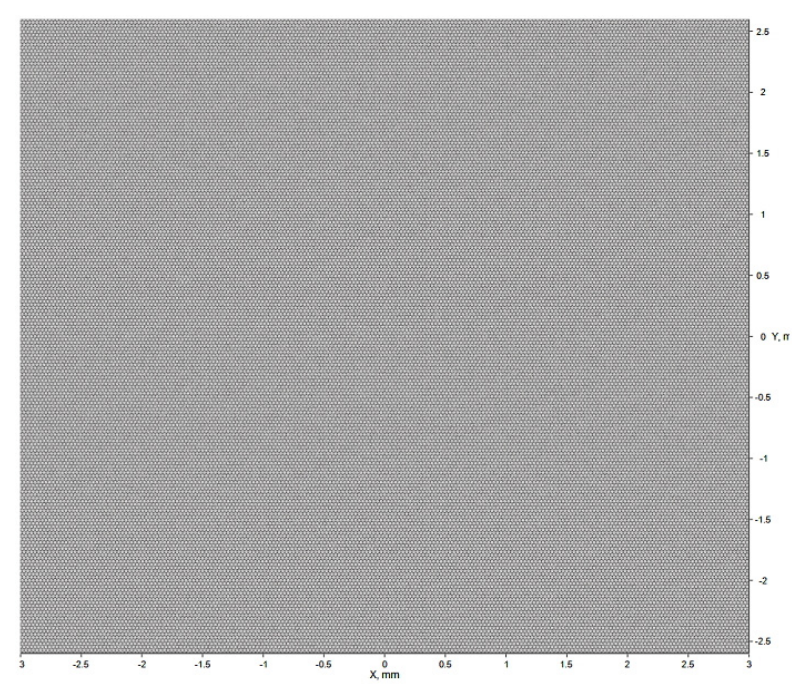

(a)

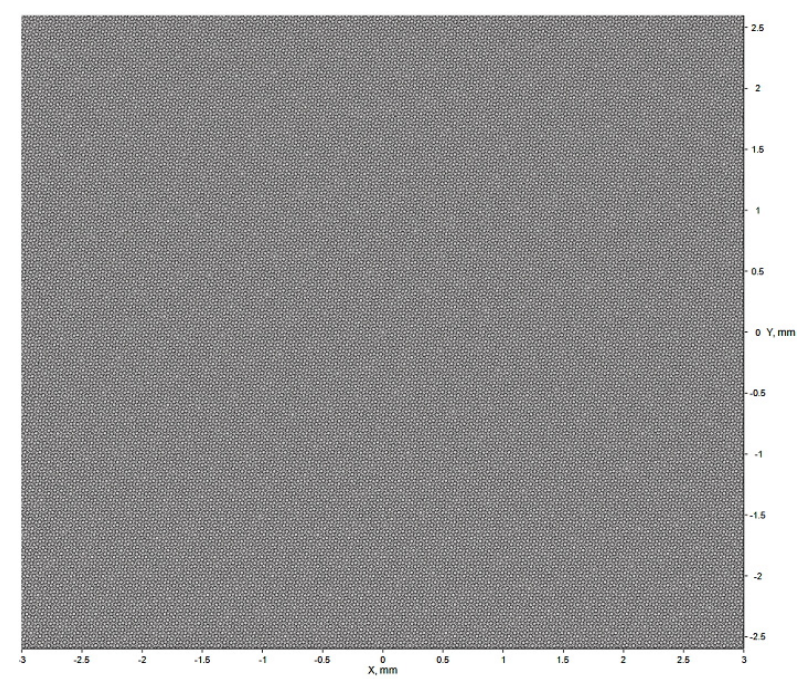

(c)

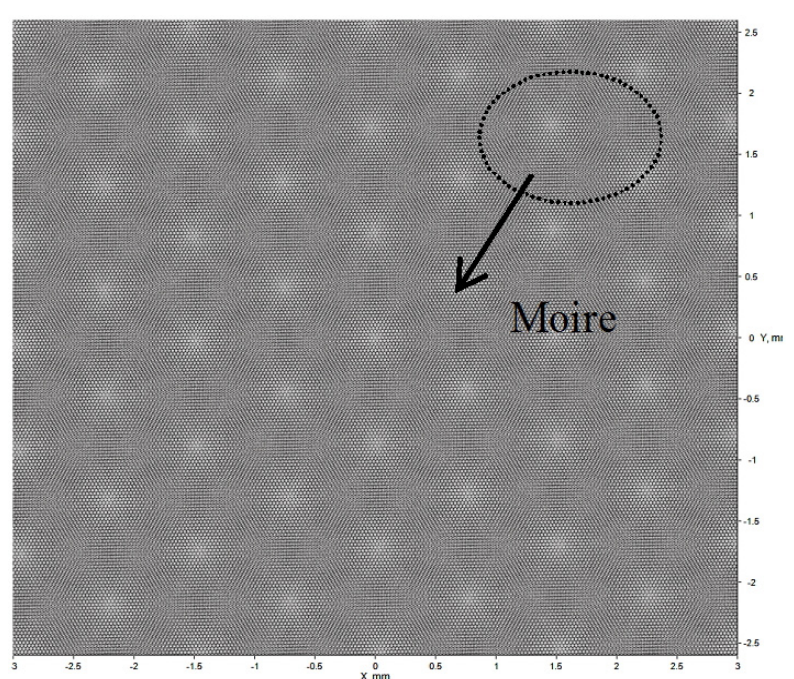

(b)

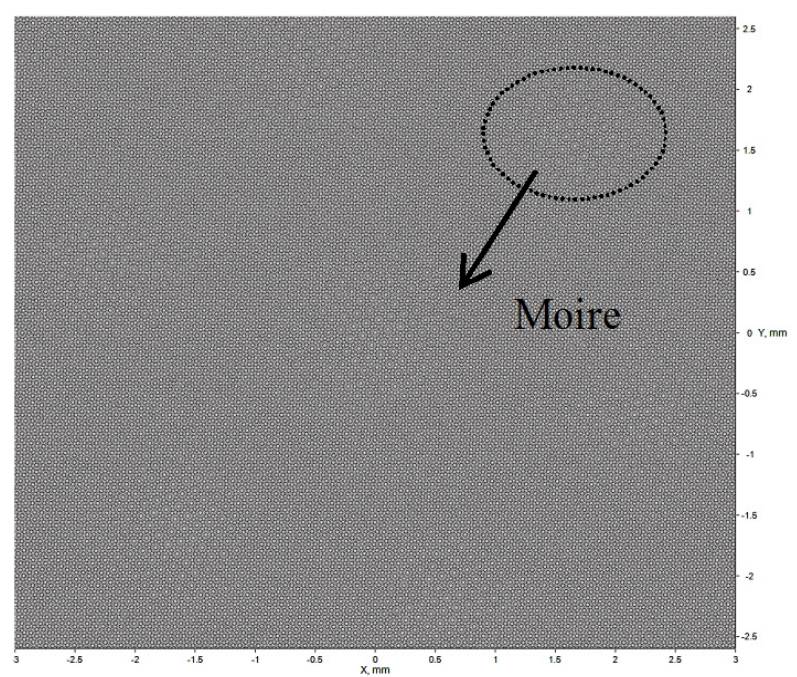

(d)

FIG. 2. Simulation results for approach 1 , where the lower film was tilted with respect to the upper film in steps of $2^{\circ}$. (a) Tilt angle $0^{\circ}$, (b) Tilt angle $2^{\circ}$, (c) Tilt angle $24^{\circ}$ (optimal), (d) Tilt angle $28^{\circ}$.

because the periodic arrangement of the microlenses still remains. Overall, this first approach is not a good solution to the moiré problem, because severe moiré fringes are formed in the vicinity of zero tilt angle, around which the period of the moiré fringe is very large.

Figure 3 shows the simulation results for the second approach, where the pitch of the holes of the lower film was adjusted between 15 and $28 \mu \mathrm{m}$. The hole diameter was set to be smaller than the pitch by $2 \mu \mathrm{m}$ for all cases. This approach is worth trying because the pitch mismatch between the two films is expected to be favorable for reducing moiré fringes. Figure 3 clearly shows that the moiré fringes become weaker as the pitch decreases, and that the moiré pattern is negligible at small pitches of 20 and $15 \mu \mathrm{m}$. However, the filling ratio decreases as the pitch increases, and the filling ratio of the film with a pitch of $15 \mu \mathrm{m}$ is only $69 \%$. This value is similar to that of a conventional
MLA film (usually $65-70 \%$ ). The filling ratio increases to $73 \%$ at a pitch of $20 \mu \mathrm{m}$, which is a little bit larger than the value for a conventional MLA film. It is thus expected that, compared to a conventional MLA film, the brightness of a backlight can be improved by adopting a hole pitch of $20 \mu \mathrm{m}$. However, there is still some possible formation of moire fringes due to the interference effect between the periodic microlenses on the MLA film and the LCD pixels.

Figure 4 shows the simulation results for the schematic of the third approach shown in Table 2, where the hole diameter was randomly adjusted in the range of 20-30 $\mu \mathrm{m}$. The average diameter was $25 \mu \mathrm{m}$. Centers of holes were arranged in a hexagonal lattice, and the tilting angle was changed by steps of $2^{\circ}$. This approach was aimed at investigating whether the degree of the moiré fringes shown in Fig. 2 could be reduced by randomizing the hole size. Figure 4 shows that although the overall moiré visibility 


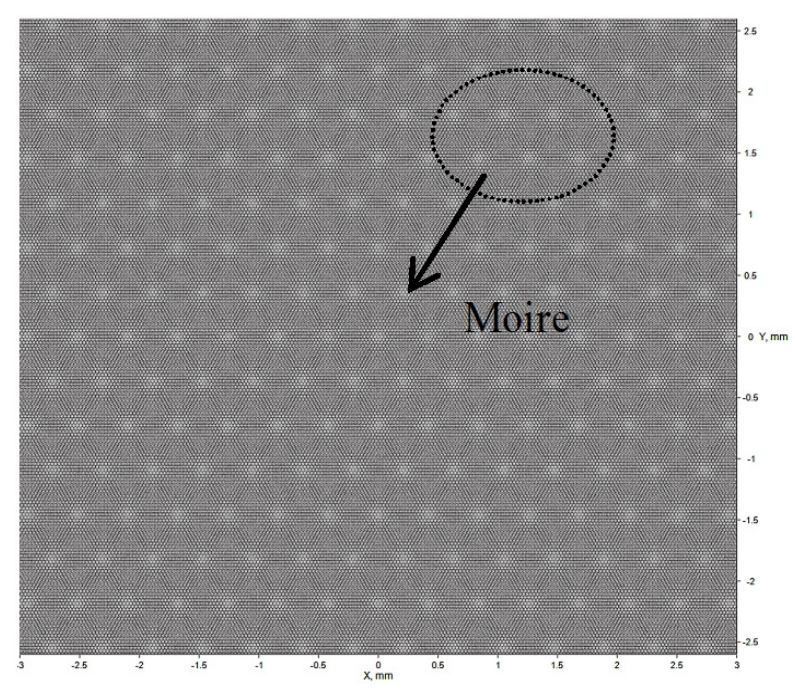

(a)

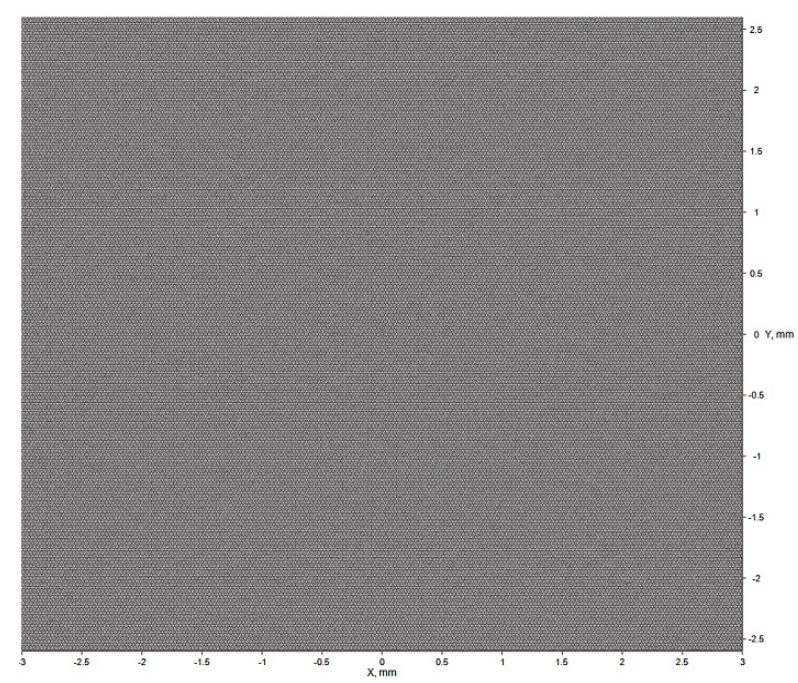

(c)

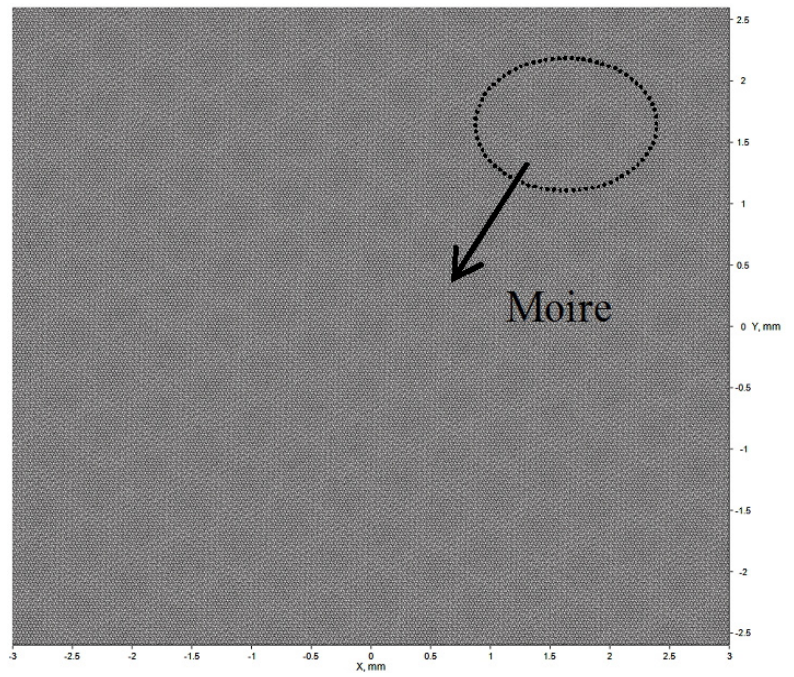

(b)

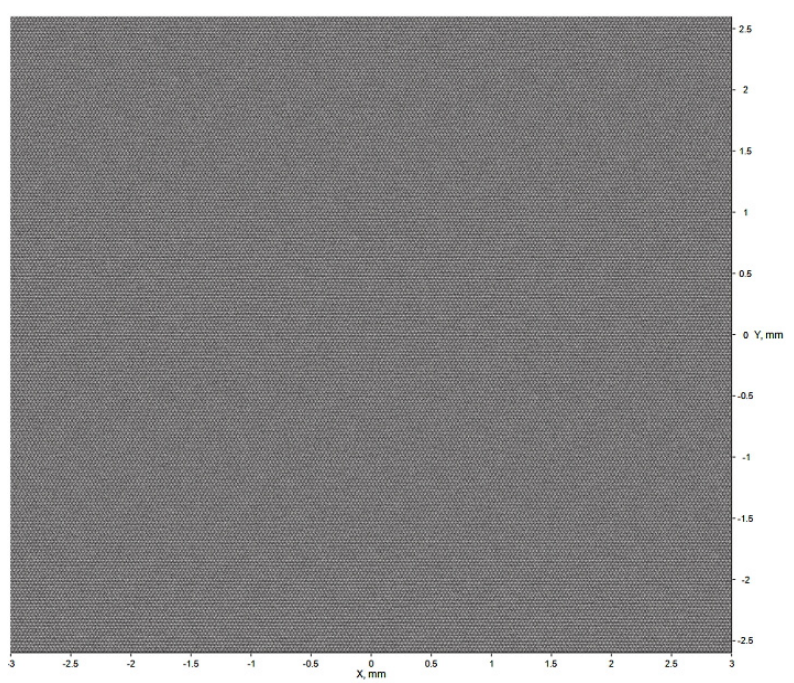

(d)

FIG. 3. Simulation results for approach 2, where the hole diameter and pitch of the lower film were changed simultaneously. (FF=Filling factor). (a) Pitch $28 \mu \mathrm{m}$, FF 78\%, (b) Pitch $24 \mu \mathrm{m}$, FF 76\%, (c) Pitch $20 \mu \mathrm{m}$, FF 73\% (optimal), (d) Pitch 15 $\mu \mathrm{m}$, FF 69\%.

becomes weaker in this approach, one can still detect moiré fringes under most simulation conditions. The fringes become weaker at a tilting angle of $10^{\circ}$. The most serious drawback of this approach is that the filling ratio is only $63 \%$, due to the decrease in the average area of holes, which is even smaller than the filling ratio of a conventional MLA film.

Figure 5 shows the simulation result for the last approach, where the hole position was randomly arranged while its diameter was fixed to be $28 \mu \mathrm{m}$. The randomization of the hole position was carried out using the sine shift algorithm, which is schematically shown in Fig. 6 [13]. According to this algorithm, the amplitude and the period of the sine modulation are adjusted to randomize the holes while keeping the filling ratio at a high value. This sinusoidal modulation was added along both $x$ and $y$ directions. The filling ratio of the optimized random pattern was $70 \%$ when the pattern diameter was $28 \mu \mathrm{m}$ and the minimum gap between adjacent holes was $1 \mu \mathrm{m}$. The optimum modulation amplitudes found for the $x$ and $y$ directions were 0.171 and $0.148 \mathrm{~mm}$ respectively, while the optimized periods were 1.142 and 0.9898 $\mathrm{mm}$. It was found that moiré fringes were hardly observed for any simulation condition, i.e. for any tilt angles. This clearly shows that the randomization of the hole positions of the lower film is an effective way to remove the moiré pattern from a stack of MLA films. Moreover, this approach has the potential to enhance the brightness of the backlight, because the filling ratio can be increased up to $81 \%$ without any moiré problems when the gap between the holes is set to be zero. Thanks to the randomized array, there is little possibility of the formation of another moiré pattern between the MLA films and the LCD pixel structure.

Finally, we would like to discuss the effect of the condition of the incident light on moiré formation. In practical cases, diffuse light is incident on the films, rather than collimated 


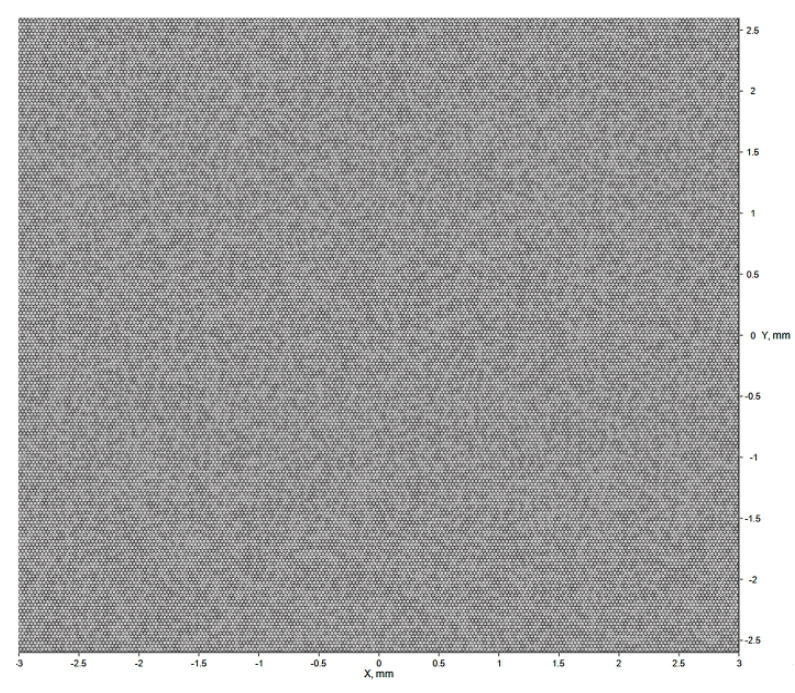

(a)

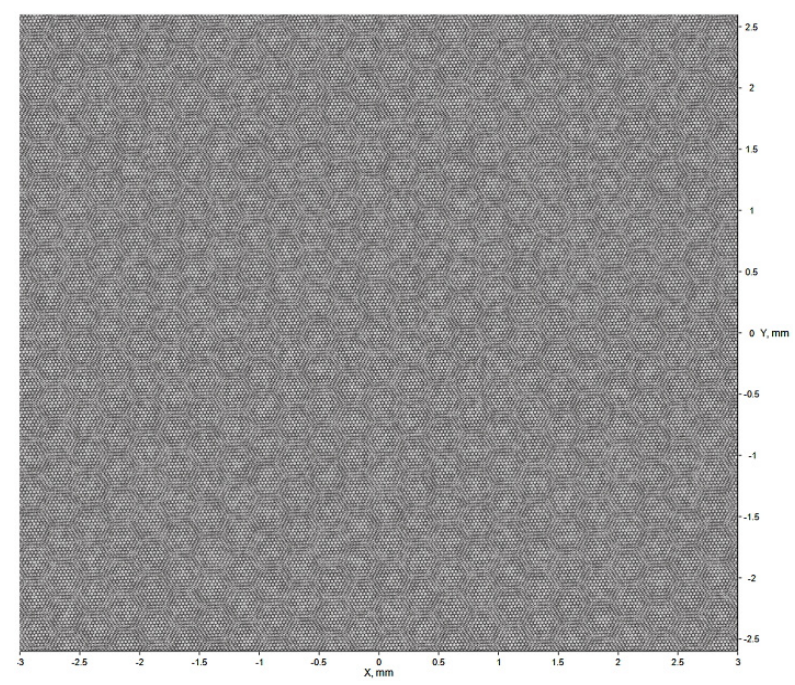

(c)

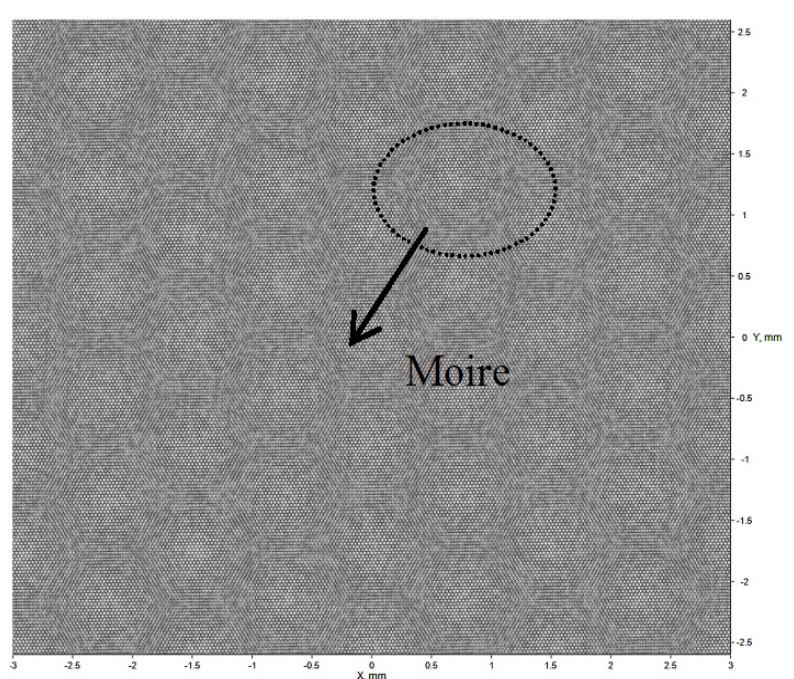

(b)

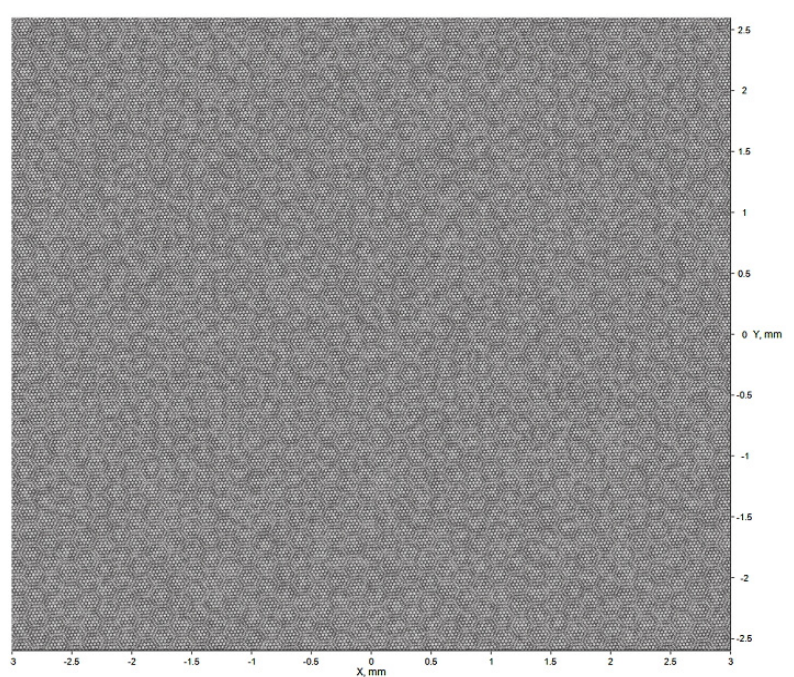

(d)

FIG. 4. Simulation results for approach 3, where the hole diameter of the lower film was changed in a certain range, and then the lower film was tilted with respect to the upper film. (a) Tilt angle $0^{\circ}$, (b) Tilt angle $2^{\circ}$, (c) Tilt angle $6^{\circ}$, (d) Tilt angle $10^{\circ}$ (optimal).

light. We carried out an additional moiré simulation based on diffuse light, which was generated using the Gaussian scattering model (diffusion angles of 0 and $5^{\circ}$ ). The results showed that the moiré pattern and its period are the same, irrespective of the diffuseness of the incident light. Only the moiré visibility shows some difference. We thus think that using collimated light in the simulation is an effective way to scrutinize the existence of the Moiré pattern due to MLA films.

\section{CONCLUSION}

Optical simulations were carried out for two stacked MLA films to find an optimized lens array for removing moiré fringes. The upper MLA film consisted of hexagonally-arranged circular holes, the filling factor of which was $79 \%$. Four different lens configurations were adopted on the lower film and investigated by optical simulation. The first approach was to tilt the lower film with respect to the upper film while the same lens configuration was used in both films. The optimum tilt angle was found to be $24^{\circ}$, but we cannot exclude the possibility that an additional moiré pattern might be generated due to the interference between the periodic lens array of MLA films and the LCD pixels. The second approach was to change the hole diameter and pitch of the lower film, which showed that the moiré fringes were minimized at a pitch of $20 \mu \mathrm{m}$ and diameter of $18 \mu \mathrm{m}$. In addition, the filling factor was $73 \%$, which was larger than for a conventional MLA film, indicating that on-axis brightness would be enhanced. However, the interference between the MLA films and LCD pixels may not be completely removed, due to the periodic lens array on the MLA films. The third approach was to randomize the hole diameter in 


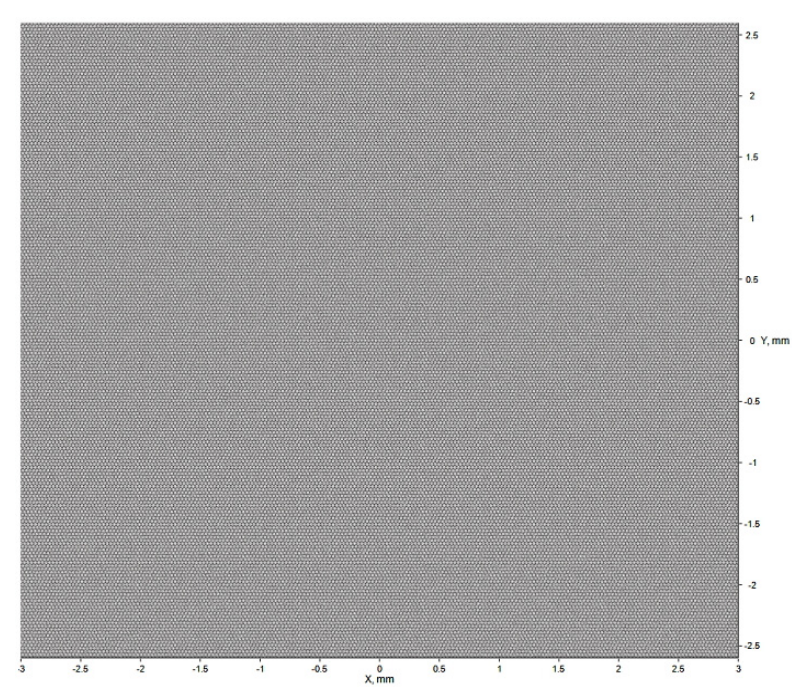

(a)

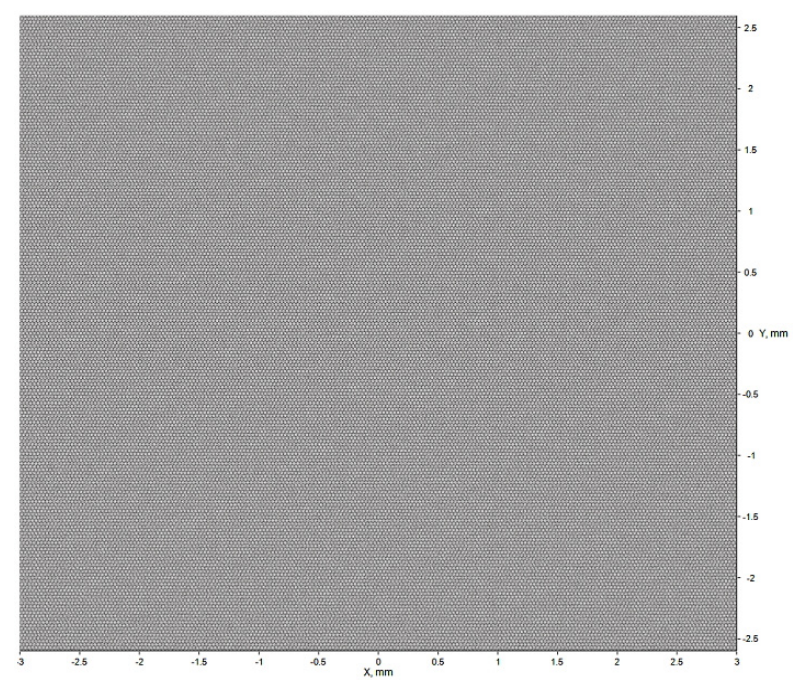

(c)

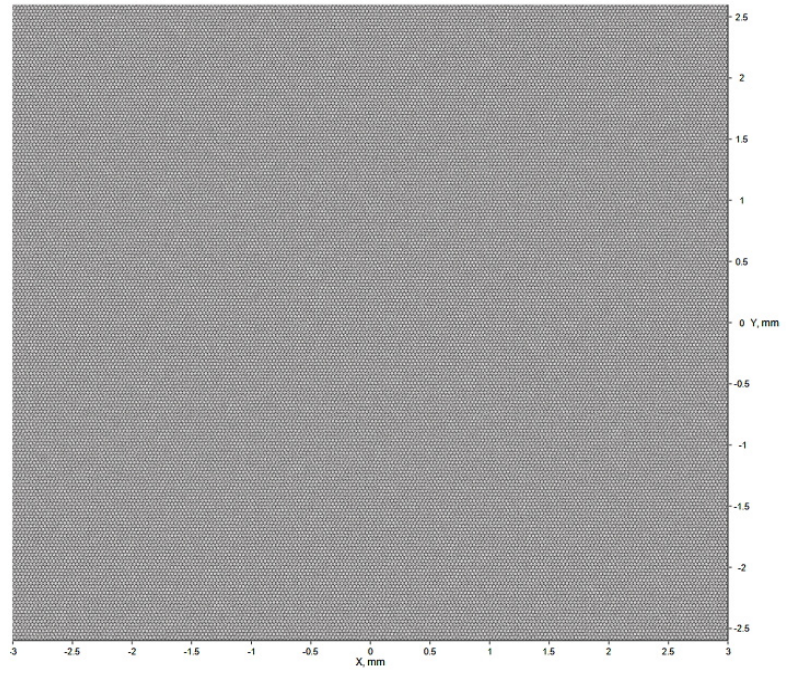

(b)

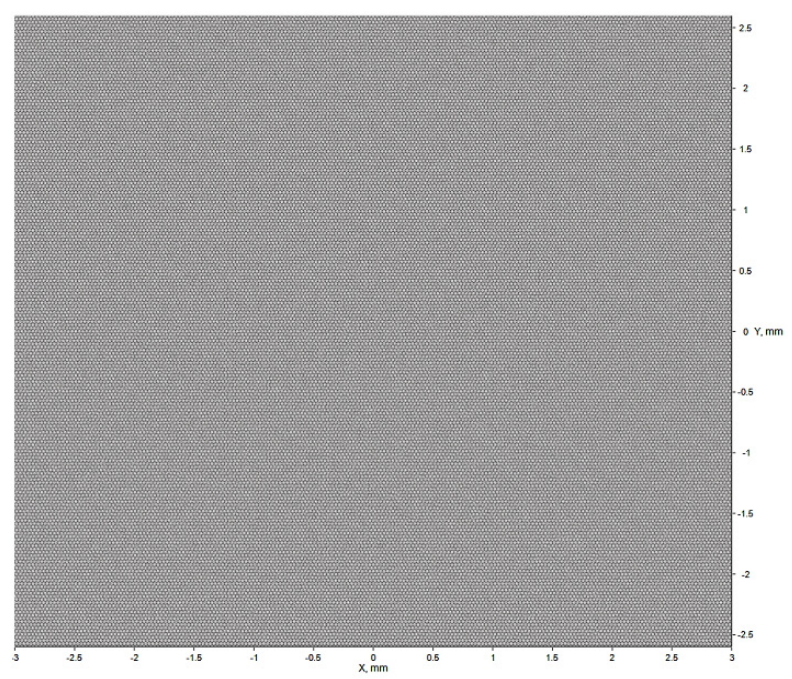

(d)

FIG. 5. Simulation results for approach 4 , where the hole positions were randomly arranged based on the sine shift algorithm. (a) Tilt angle $0^{\circ}$, (b) Tilt angle $6^{\circ}$, (c) Tilt angle $14^{\circ}$, (d) Tilt angle $30^{\circ}$.

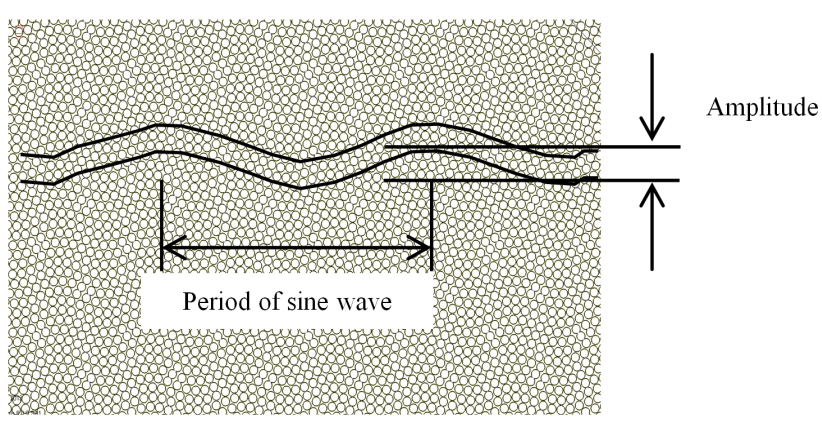

FIG. 6. A schematic view of the randomized hole array generated using the sine shift algorithm.

a certain range, which was found to be effective in reducing the moiré fringes under all conditions. However, the filling factor was very low, suggesting there is no advantage of this design over a conventional film, from the viewpoint of efficacy. The last approach was to randomize the lens positions while keeping the filling factor at a high value, above $70 \%$. The holes were randomly arranged according to the sine shift algorithm, and it was found that the moire fringes were effectively removed, thanks to the random configuration of the microlenses. The filling factor was $70 \%$ when the minimum distance between holes was 1 $\mu \mathrm{m}$, and may be increased to $81 \%$ if this gap is set to zero. Moreover, an additional interference pattern between the MLA films and LCD pixels may not occur, thanks to the randomized configuration of microlenses. This study suggests that randomizing the lens configuration while keeping the filling factor high is an effective way to develop high-gain MLA films for backlight applications. 


\section{ACKNOWLEDGMENT}

This research was financially supported by the Ministry of Education (MOE) and National Research Foundation of Korea (NRF) through the Human Resource Training Project for Regional Innovation (No.2014029502) and by the Hallym University Research Fund, 2012 (HRF-G-2012-3).

\section{REFERENCES}

1. M. Tjahjadi, G. Hay, D. J. Coyle, and E. G. Olczak, "Advances in LCD backlight film and plate technology," Information Display 10/06, $22-27$ (2006).

2. G.-J. Park, Y.-G. Kim, J.-H. Yi, J.-H. Kwon, J.-H. Park, S.-H. Kim, B.-K. Kim, J.-K. Shin, and H.-S. Soh, "Enhancement of the optical performance by optimization of optical films in direct-illumination LCD backlight," J. Opt. Soc. Korea 13, 152-157 (2009).

3. J. S. Seo, T. E. Yeom, and J.-H. Ko, "Experimental and simulation study of the optical performances of a wide grid polarizer as a luminance enhancement film for LCD backlight applications," J. Opt. Soc. Korea 16, 151-156 (2012).

4. H. Ottevaere, R. Cox, H. P. Herzig, T. Miyashita, K. Naessens, M. Taghizadeh, R. Volkel, H. J. Woo, and H. Thienpont, "Comparing glass and plastic refractive microlenses fabricated with different technologies," J. Opt. A: Pure
Appl. Opt. 8, S407-S429 (2006).

5. C. T. Pan and C. H. Su, "Fabrication of gapless triangular micro-lens array," Sens. Actuators A 134, 631-640 (2007).

6. S. K. Hwang, S. H. Baek, J. H. Kwon, and Y. S. Park, "Fabrication of microlens array using photoresist thermal reflow," Korean J. Opt. Photon. (Hankook Kwanghak Hoeji) 20, 118-122 (2009).

7. K. M. Baker, "Highly corrected close-packed microlens arrays and moth-eye structuring on curved surfaces," Appl. Opt. 38, 352-356 (1999).

8. S.-I. Chang, J.-B. Yoon, H. Kim, J.-J. Kim, B.-K. Lee, and D. H. Shin, "Microlens array diffuser for a light-emitting diode backlight system," Opt. Lett. 31, 3016-3018 (2006).

9. B.-Y. Joo and D.-H. Shin, "Simulations of pixel moirés in the liquid crystal display with image processing technique," Displays 30, 190-194 (2009).

10. S.-J. Byun, S. Y. Byun, J. Lee, W. M. Kim, H.-P. Kim, M. Y. Jeon, and T.-S. Lee, "An efficient simulation and analysis method of moiré patterns in display systems," Opt. Express 22, 3128-3136 (2014).

11. E. G. Olczak, M. Yamada, D. J. Coyle, and D. R. Olson, "A Moiré-free platform for LCD backlighting," in Proc. SID Tech. Digest (San Francisco, USA, Jun. 2006), pp. 13361339.

12. B.-Y. Joo and J.-H. Ko, "Analysis of color uniformity of white LED lens packages for direct-lit LCD backlight applications," J. Opt. Soc. Korea 17, 506-512 (2013).

13. Optimization Module User's Guide (LightTools v8.1, Synopsys, 2013). 